\title{
Searching for Evidence of Optical Rectification: Optically-Induced Nonlinear Photovoltage in a Capacitor Configuration
}

\author{
Somayeh M. A. Mirzaee, ${ }^{1}$ Jean-Michel Nunzi, ${ }^{1, *}$ \\ ${ }^{1}$ Department of Physics, Engineering Physics and Astronomy and Department of Chemistry, Queens University, Kingston, Ontario \\ K7L 3N6, Canada \\ *Corresponding author: nunzijm@queensu.ca
}

Received XX Month XXXX; revised XX Month, XXXX; accepted XX Month XXXX; posted XX Month XXXX (Doc. ID XXXXX); published XX Month XXXX

\begin{abstract}
A current challenge in photonics is to design new versatile photodetectors based on optical rectification induced photo-voltage; these ones are more attractive than classical photodetectors because they do not rely on band to band transitions. Identification of the origin of the photovoltage detected under intense illumination can sometimes be confusing due to the competition between several nonlinear processes. Examples of such processes are optical rectification, multiphoton absorption, and photothermal heating, all of which may result in the detection of a DC photovoltage in a capacitor configuration. Herein, differences between the resulting photovoltage from these processes are analyzed, and techniques are proposed to distinguish between optical rectification-induced DCphotovoltage and the photovoltage resulting from alternative effects.
\end{abstract}

OCIS codes: (190.4360) Nonlinear optics, devices; (190.4400) Nonlinear optics, materials; (190.4350) Nonlinear optics at surfaces; (190.4870) Photothermal effects.

http://dx.doi.org/10.1364/optica.99.099999

\section{INTRODUCTION}

Applications ranging from energy conversion to photodetection are critically related to how electromagnetic radiation is collected and converted into direct current. However, there are still lots of challenges related to the development of a broadband device that could convert electromagnetic radiation into electrical energy, spanning from the infrared (IR) through to the visible spectrum.

Optical rectification (OR) is a second-order nonlinear optical process that is responsible for the generation of DC polarization in nonlinear media. This process has been used in many recent reports to convert light directly into DC voltage $[1,2,3,4,5,6]$. The ultrafastresponse time of the second-order nonlinear OR and its broadband response spectrum at optical frequencies make it a good candidate for the development of next generation energy conversion devices [7] that can overcome present limitations of semiconductor photovoltaic cells. The remarkable properties of plasmon enhanced OR effect can lead to possible applications in room-temperature IR-photodetectors, which do not suffer from the same limitations as current technologies, such as high noise level, slow response time, and spectral restrictions [8]. In the near future, optical rectification may also be combined with optical nanoantenna to build efficient solar energy converters: the so-called 'rectenna' solar-cells $[9,10,11,12]$.

However, OR-based applications will not be efficient without enhancing the OR process, which is not achievable without a precise knowledge of the source and nature of the contributions. The optical rectification process generates a bound charge distribution which is detectable only in a capacitive coupling. There have been several reports of induced DC photovoltage in a capacitor configuration that is interpreted as the nonlinear OR process $[13,14,15]$. However, theoretical studies [16], as well as our own experimental investigations [17], show that in most cases OR cannot be the true explanation for DC photocurrent that has been detected in a capacitor configuration. Plasmon absorption [18], nonlinear multiphoton absorption [17], and built-in potential due to work function differences [19] are some possible artifacts providing alternative explanations.

In this paper, we present a method and the related apparatus to study the differences between the generated photovoltage through optical rectification process and some other nonlinear processes in thin films. The method effectiveness is examined through three different experimental conditions: the first experiment dealing with the multiphoton absorption (MPA) process as the dominant nonlinear process in samples under study, in the second experiment the thermally-induced work function variations is the dominant process, 
and in the third one the hot-electron injection originated OR process is designed to be the predominate process. Our experiments open directions to revisit some of the previously reported experimental results.

\section{OPTICAL RECTIFICATION BACKGROUND}

In the framework of nonlinear optics, the relationship between the induced polarization $(\mathrm{P})$ in a lossless and dispersion-less medium and the incident electric field strength (E) is described as follows:

$$
\begin{gathered}
P_{i}=\varepsilon_{0}\left(\chi_{i j}^{(1)} E_{j}+\chi_{i j k}^{(2)} E_{j} E_{k}+\chi_{i j k l}^{(3)} E_{j} E_{k} E_{l}\right. \\
\left.\ldots+\chi_{i j k l m n}^{(5)} E_{j} E_{k} E_{l} E_{m} E_{n}+\ldots\right) .
\end{gathered}
$$

In which $\chi^{(\mathrm{n})}$ is an n-rank tensor that is the $\mathrm{n}^{\text {th-order }}$ optical susceptibility and $\varepsilon_{0}$ is the permittivity of vacuum. The lowercase Latin alphabet subscripts denote the Cartesian coordinates which according to the Einstein notation are summed-up when repeated in a product. For example, consider a polar crystal with nonzero second-order susceptibility $\chi^{(2)}$ being illuminated by a laser beam with polarization along the $c$-axis whose electric field strength is represented as $E(t)=E_{0} e^{-i \omega t}+c . c .$. Equation 1 gives the generated second-order nonlinear polarization along the $c$-axis in the crystal as: $P^{(2)}(t)=2 \varepsilon_{0} \chi^{(2)} E E^{*}+\left(\varepsilon_{0} \chi^{(2)} E^{2} e^{-i 2 \omega t}+c . c.\right)$. It consists in a static component at zero frequency, plus another one oscillating at frequency $2 \omega$. The latter component is the source term for radiation at second-harmonic frequency. As the second time derivative of the first component vanishes, it does not lead to the generation of an electromagnetic radiation in the DC regime; instead, it induces a static electric field across the nonlinear crystal. This is known as optical rectification (OR). As seen in Eq. 1, the DC polarization of the material depends on the square of the incident electric field. Considering the firstorder in $\chi^{(2)}$, a static charge distribution will be created inside the nonlinear material, which will be linearly proportional to the polarization induced by OR. Since the induced charge is a surface charge which remains bound, only capacitive coupling will make it possible to detect the OR signal as a photovoltage inside a material or structure. A capacitor configuration can be used as shown in Fig. 1. The induced voltage across the electrodes can be expressed as $V \propto \rho \propto \chi^{(2)} 2\left|E_{0}\right|^{2}$, where $\rho$ is the static charge distribution inside the material [16].

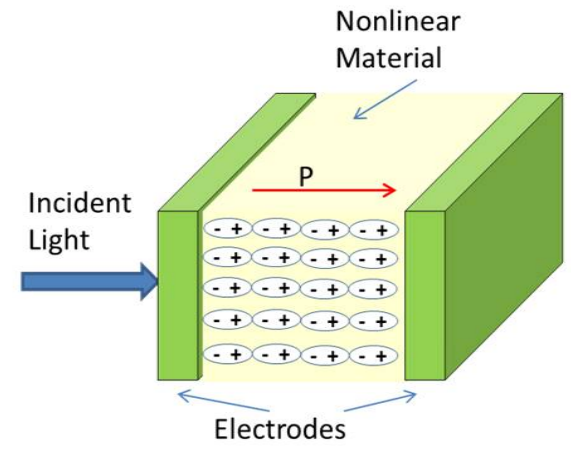

Fig. 1. Capacitor configuration to detect $\mathrm{OR}$ in the nonlinear material.

\section{EXPERIMENTS AND DISCUSSIONS}

Since the optical rectification process is naturally weak for a thin film and practically impossible to detect in the case of a continuous-wave opticalillumination [16], we used a chopped continuously mode-locked femtosecond laser with about $30 \mathrm{~kW}$-peak power. However, in such excitation scheme there is a strong possibility to trigger other nonlinear photovoltage-generating mechanisms. In the remainder of this paper, we focus on the characteristic analysis of the OR-process and its differences with some similarly induced nonlinear DC-photovoltages in the capacitor configuration. Although not the entire manifold of nonlinear effects can be addressed in this study, it allows shedding some light on effects that may and do influence the detection of OR-induced photovoltage.

\section{A. Induced Photovoltage via Bulk Multiphoton Absorption (MPA)}

One of the possible processes that may generate photovoltage in a medium under an intense femtosecond laser is nonlinear multiphoton absorption (MPA). Inspired by previous experiments on organic photodetectors in our group, we performed MPA measurements with ultrafast laser pulses illuminating a nonlinear semiconducting polymer device. Our goal was to clarify the MPA signal characteristics in order to distinguish it from OR signal.

The devices were fabricated using a transparent indium tin oxide (ITO) coated glass electrode as the substrate. A $120 \mathrm{~nm} \mathrm{Ag-thin} \mathrm{film} \mathrm{was}$ used as the top electrode. The active layer of the photodiode was a thin film of a P3HT:PCBM bulk-heterojunction. A schematic view of the devices is shown in Fig. 2-inset. The nonlinear medium chosen was P3HT, which is a widely explored semiconducting polymer in research involving organic field effect transistors, solar cells [20], organic light emitting diodes [21], nonlinear optical devices [22], and flexible IR detectors [23]. Devices with slightly different fabrication procedures were investigated at this step to evidence the two- and three-photon absorption processes.

The absorption spectrum of the P3HT/PCBM layer shows a peak at about $500 \mathrm{~nm}$, which is a characteristic of P3HT, and negligible absorption in the near infrared region.A three-photon absorption(3PA) resonance occurs at wavelengths three times longer than the singlephoton absorption peak, which creates favorable conditions to observe a photovoltage generated in the cells at $1550 \mathrm{~nm}$. The mechanism of photovoltage generation in a donor-acceptor junction is already explained in Ref. [20]. A custom-built photovoltage scanning apparatus, shown schematically in Fig. 2, was used for data acquisition. The apparatus allows us to run polarization studies over the generated photovoltage, simultaneously along with the second and third harmonic generation (SHG, THG) signals. The samples were illuminated with the laser source-generating polarized light pulses centered at $1550 \mathrm{~nm}$ wavelength. The sample was placed at the focal plane of a focusing lens. The photodiode generated voltage versus the input illumination power is shown in Fig. 3(a). Different samples show slightly different photoresponses; these differences are attributed to the fabrication and annealing conditions as well as to aging of the samples.

As it is shown, the slope of the experimental data is very close to 3 decades of photovoltage per decade of optical power in a log-log scale, which can be described by Eq. 1 . There, it is clear from the time averaging that only the odd orders of polarization can contribute to the absorption process under a monochromatic field. Consequently, a three-photon absorption process is proportional to the fifth-order nonlinear polarization and the absorbed energy is proportional to the cube of the light intensity. Furthermore, the 3PA photocurrent from Fig. 3(a) can be correlated with the short circuit current (USC) delivered by the same samples under $100 \mathrm{~mW} / \mathrm{cm}^{2}$ illumination from a solar 
simulator. The results are represented in Table.1 of Ref. [17], which show that $J_{S C}$ follows the same trend as the 3PA short-circuit (J3PA) photocurrent.

Thus, checking the dependency of the output photovoltageversus the pump power can be considered as the simplest measurement test for any multiphoton absorption source versus the optical rectification one.

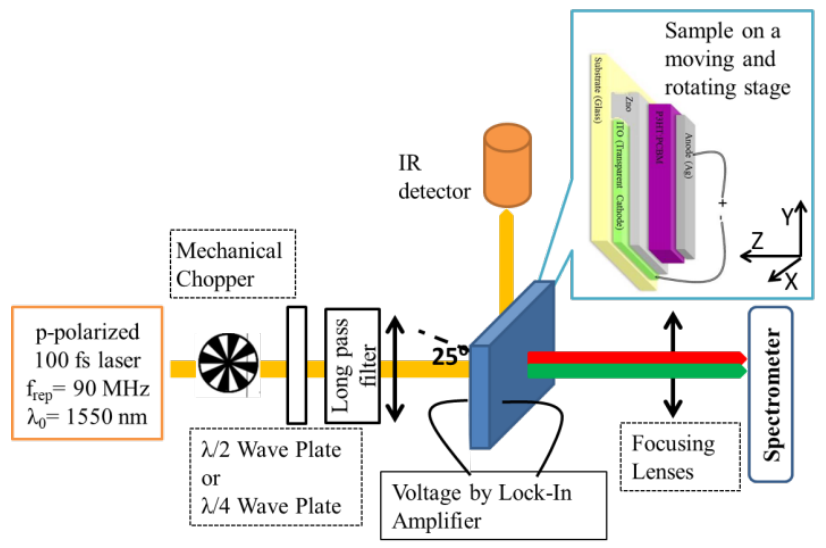

Fig. 2. The measurement setup.

What can we conclude if the dependency of the output photovoltage versus the pump power is not explainable by the bulk absorption spectrum? To explain this situation, our next set of experiments reveals additional evidence about the multiphoton absorption process and its differences from the OR mechanism.

\section{B. Induced Photovoltage via Deep States Multiphoton Absorption}

Another possibility to study multiphoton absorption is through the deep trap absorption of the P3HT blend, which is different from the bulk absorption. Various reports confirm the presence of localized states within the band gap [24,25]. Some variations in photodiode fabrication conditions, like the presence of oxygen, can increase the density of available deep traps. Figure $3 \mathrm{~b}$ shows the evolution of the generated photovoltage versus light power for newly fabricated photodiodes. The slope of the curve is very close to 2 decades of photovoltage per decade of optical power in a log-log scale, which shows that the dominant MPA in these cells is two-photon absorption (TPA). The TPA behavior of photodiodes can be explained by the localized states within the band gap at interfaces [26]. However, to confirm that these observations are due to the actual TPA and not to other possible photo-generation events, we investigate the effects of the polarization state of the incident light on the intensity of the detected signal.

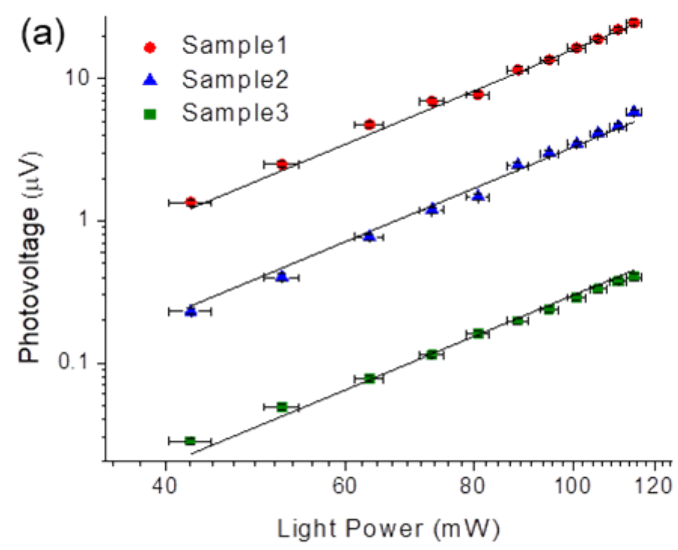

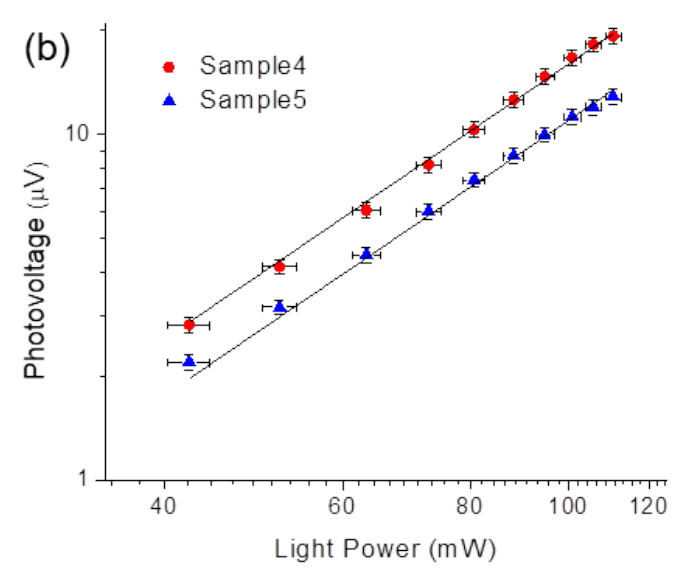

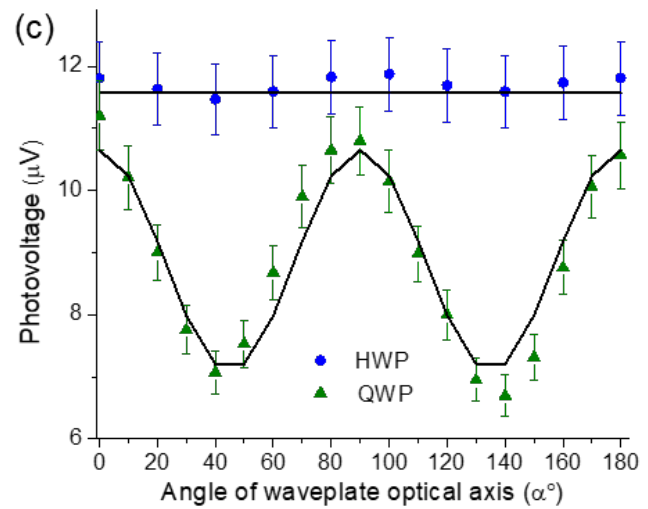

Fig. 3. (a) 3PA photovoltage for three differentsamples under study. (b) TPA photovoltage for two different samples under study. (c) Polarization dependence of the TPA photovoltage. The points show the experimental data and the solid lines correspond to the 3PA, TPA, linear and $2+\cos ^{2}(2 \alpha)$ models in (a), (b), (c) half-wave-plate (HWP) data, and (c) quarter-wave plate (QWP) data, respectively.

The TPA photoresponse to the polarized light shows almost a constant dependence versus linear polarization and some oscillatory dependence versus circular polarization, as seen in Fig. 3(c). Small variations from the linear fit in Fig. 3(c) for linearly polarized light is just due to the change in Fresnel coefficients at the air-glass interface with a maximum at p-polarized light. It has been identified that the measured TPA photovoltage for the circularly polarized incident light versus the linearly polarized one is very close to the ratio of $2 / 3$. This ratio is expected for an isotropic nonlinear material [5]. Therefore, polarization study is another meaningful analysis to distinguish between MPA and OR-photovoltages. However, this is not always a feasible test due to the experimental limitations like the coupling of surface plasmon [13].

\section{Thermally-induced Photovoltage}

To proceed with the investigation on similar photovoltages to OR photosignal, we considered thermal effects in a capacitor configuration. In capacitor devices, there is a possibility to detect photosignal generated through changes in the relative work functions (WF) of the electrode surfaces. Work function of a given surface depends on the surface's Fermi level and the electrostatic potential in the vacuum near 
the surface. Therefore, the space between two dissimilar conductor surfaces will experience a built-in electric field, which can be measured by different technique [27]. However, even for two similar electrodes, as soon as one of the pair's WF changes, a potential difference will become detectable [19].

Metal's WF variations versus temperature are a consequence of several mechanisms, such as thermal expansion, atomic vibration, chemical potential variation, spontaneous volume change [28], and oxygen adsorption [29].

To better understand light-induced heating and its related WFvariations in a capacitor configuration, we performed an experiment on a gold thin film. The prepared sample was similar to samples used in earlier OR detection experiments [13]. A $20 \mathrm{~nm}$ thick gold thin film deposited directly on an ITO substrate and sandwiched in the capacitor geometry used for photovoltage measurements as is shown in Fig. 4(b).

The sample is illuminated by the femtosecond source, as seen in the setup shown in Fig. 2, away from the focal point to intentionally reduce the chance of generating OR nonlinearity. The measured photovoltage was a few microvolts (less than $10 \mu \mathrm{V}$ ). A polarization study on the signal shows no changes while the polarization status is changing linearly or elliptically. In addition, the detector connected to the spectrometer shows almost zero SHG and THG signals. These results are in contradiction to the specifications of the OR-induced photovoltage corresponding to the nonvanishing nonlinear susceptibility tensor elements at the gold's surface. However, based on the observed photovoltage characteristics, the mechanism that most explains the detected photovoltage is a thermally activated process.

The laser-induced temperature variation on the sample and its related WF changes can be estimated as follows: the surface temperature rises $\Delta T(t)$ with the heat caused by a square-shaped laser pulse of duration $t_{0}$ calculated using the procedure from D. Burgess etal. [30].

$$
\begin{aligned}
\Delta T(t) & =2\left(\frac{F_{0}}{K}\right)\left(\frac{k}{\pi}\right)^{\frac{1}{2}} \quad\left(t<t_{0}\right), \\
& =2\left(\frac{F_{0}}{K}\right)\left(\frac{k}{\pi}\right)^{\frac{1}{2}}\left[t^{\frac{1}{2}}-\left(t-t_{0}\right)^{\frac{1}{2}}\right] \quad\left(t>t_{0}\right) .
\end{aligned}
$$

Where, Kand $k$ are thermal conductivity and diffusivity, respectively, $t_{0}$ is the laser pulse duration, and $F_{0}$ is the maximum absorbed laser power density $\left(\mathrm{MW} / \mathrm{cm}^{2}\right)$ calculated as:

$$
F_{0}=\left(P_{\text {in }}-P_{\text {out }}\right) / A, \frac{P_{\text {out }}}{P_{\text {in }}}=\exp (-\alpha X),
$$

where $P_{\text {in }}$ and $P_{\text {out }}$ are the incident and transmitted light powers for each individual layer of the sample, correspondingly. $\alpha$ is the absorption coefficient, $X$ is the layer thickness, and $A$ is the area of exposure, which was about $10^{-4} \mathrm{~cm}^{2}$.

Table 1. ITO [31] and gold [32, 33, 34,35] thermal coefficients

\begin{tabular}{c|c|c|c} 
Material & $\mathrm{K}(\mathrm{w} / \mathrm{cm} \cdot \mathrm{K})$ & $\mathrm{k}\left(\mathrm{cm}^{2} / \mathrm{s}\right)$ & $\begin{array}{c}\alpha\left(\mathrm{cm}^{-1}\right) \text { at } 1.5 \\
\mu \mathrm{m}\end{array}$ \\
\hline ITO coating & 0.04 & 0.012 & 35000 \\
\hline \multirow{2}{*}{ Gold } & $\begin{array}{c}3.17 \text { (bulk) } \\
1(20 \mathrm{~nm} \text { thin } \\
\text { film) }\end{array}$ & $\begin{array}{c}1.29(\mathrm{bulk}) \\
15(20 \mathrm{~nm} \\
\text { thin film })\end{array}$ & 867320
\end{tabular}

The absorption coefficient of the substrate's ITO layer is calculated based on transmission experiments carried at $1.5 \mu \mathrm{m}$ with the bare ITO glass. Using the laser peak power of about $30 \mathrm{~kW}$, the solutions to equations 2 and 3 yield temperature changes of $110 \mathrm{~K}$ and $56 \mathrm{~K}$ in the ITO and gold layers, respectively. In total, the temperature variations of the ITO/gold electrode for a laser pulse should be about $166 \mathrm{~K}$.

According to literature, the WF variation rate depends on the material, as different procedures are involved in each case. Published values show that the work function of gold may change as much as 0.7 $\mathrm{eV}$ at $770 \mathrm{~K}$ due to high oxygen absorption at high temperatures [29]. However, a different mechanism suggests that the work function of silver changes about $0.2-0.3 \mathrm{eV}$ at $700 \mathrm{~K}$ [36]. These numbers suggest that the work function of the electrode consisting of a thin gold layer on top of ITO glass will decrease by a couple of tenths of an electron volt for a one-hundred-degree temperature increment.

The capacitance of the capacitor shown in Fig. 4(b) was measured using a BK Precision LCR/ESR-meter, model 885 to be about $70 \mathrm{Pf}$. Considering the fraction of the illuminated area to the total area of the capacitor, the detectable photovoltage, relative to work function changes of about one-tenth of an electron volt, would be in the $\mu \mathrm{V}$-range. The calculated photovoltage through the thermal effect is in total agreement with the experimentally detected photo signal.

Based on the above discussions on different capacitor configurations, we argue that several reported optical rectification signals could have just been a misinterpretation of the experimental results. In most cases, owing to experimental setup limitations, there was no possibility to check for the validity of the reported claims.

Other examples of electro-optical effects in a metallic thin film that can generate sensible photovoltage in a capacitor configuration are: the photon-drag effect [37,38], current injection [39], drift current [40], and photo-Dember effect $[41,42]$. However, most of these effects are not relevant to the response of the capacitor structure under study.

\section{Induced Photovoltage via the Optical Rectification Process at the Metal-Semiconductor Interface}

In this section, we apply the same methodology as in previous sections to study the OR generated photovoltage via a nanostructured metallic thin film. Despite the large number of reports on metal surface and bulk contributions to SHG $[43,44]$, there are notso many reports on studying the optical rectification process in nanostructured metallic thin films [45], and to our knowledge no OR voltage could be detected for bare metallic thin films.

In our study, several silver and gold thin films were prepared on ITO substrates in order to optimize the process and parameters for generating a plasmonic nanostructure with significant absorption at $1550 \mathrm{~nm}[46,47,48,49]$. The optical absorption spectrum and scanning electron microscope (SEM) image of a $14 \mathrm{~nm}$ gold thin film are shown in Figs. 4(a) and 4(b), respectively. The broadened absorption spectrum covering the near-infrared (NIR) region plus the SEM image confirm that the rough thin film consists of randomly placed isolated Au nanoislands with strong interparticle interaction between neighboring particles $[50,51]$. 
(a)

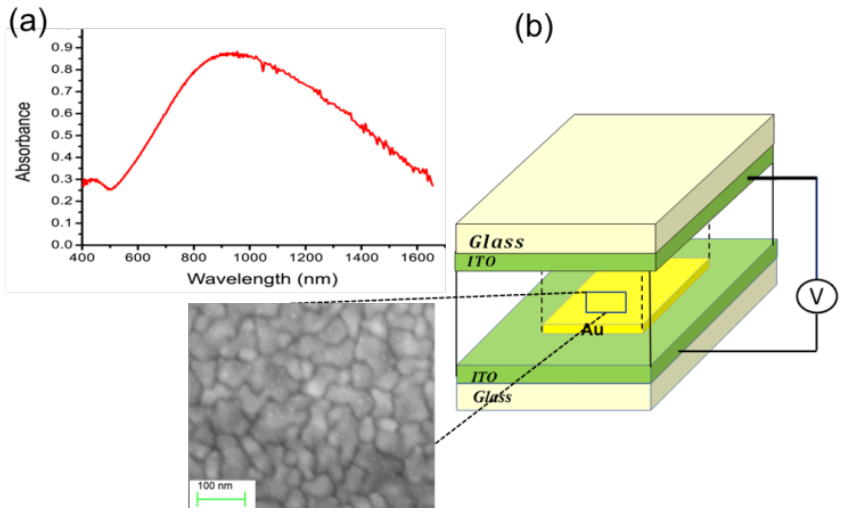

Fig. 4. $14 \mathrm{~nm}$-thick gold film deposited on top of ITO substrate (a) Optical absorption spectrum (b) Capacitor configuration incorporating the gold sample. The inset shows a scanning electron microscopy (SEM) image of an Au-thin film.

A capacitor device built from the gold sample in the same configuration represented in Fig. 4(b) was used for photovoltage measurements. The dependency of the output photovoltage versus the illumination power was tested as the first suggested measurement in section 3-A, to identify any sources of OR photovoltage. The result as illustrated in Fig. 5 shows a linear dependence on the laser source irradiance, which is expected for an OR induced photovoltage as described by Eq.1.

The polarization dependence of OR, THG, and SHG signals under $1550 \mathrm{~nm}$ illumination is illustrated in Fig. 6. To investigate the nonzero components of the second- and third-order nonlinear susceptibility tensors, each signal is plotted as a function of the incident linear polarization direction.

Simultaneous observations of OR photovoltage, SHG and THG signals show that the sample is very close to the focal point of the laser. This positioning at the highest light intensity allows for the detection of different nonlinear optical effects from the sample. The photovoltage variation versus linear polarization confirms that the voltage is neither due to multiphoton absorption nor to thermal processes. The physical mechanism responsible for the measured photovoltage can be explained via the polarization activities seen in Figs. 6(a) and 6(b).

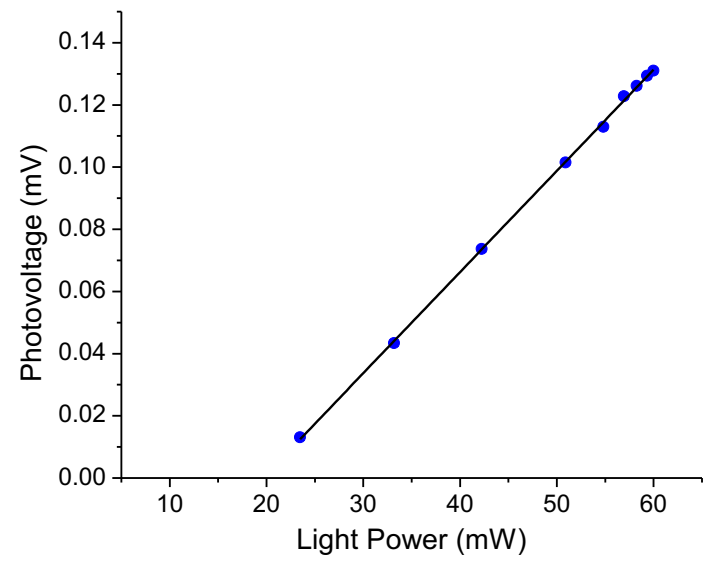

Fig. 5. Photovoltage measurement showing dependency on the illuminated laser power, with the linear fit denoted as a black line. (a)

Angle between incident polarization and HWP optical axis ( ${ }^{\circ}$ )
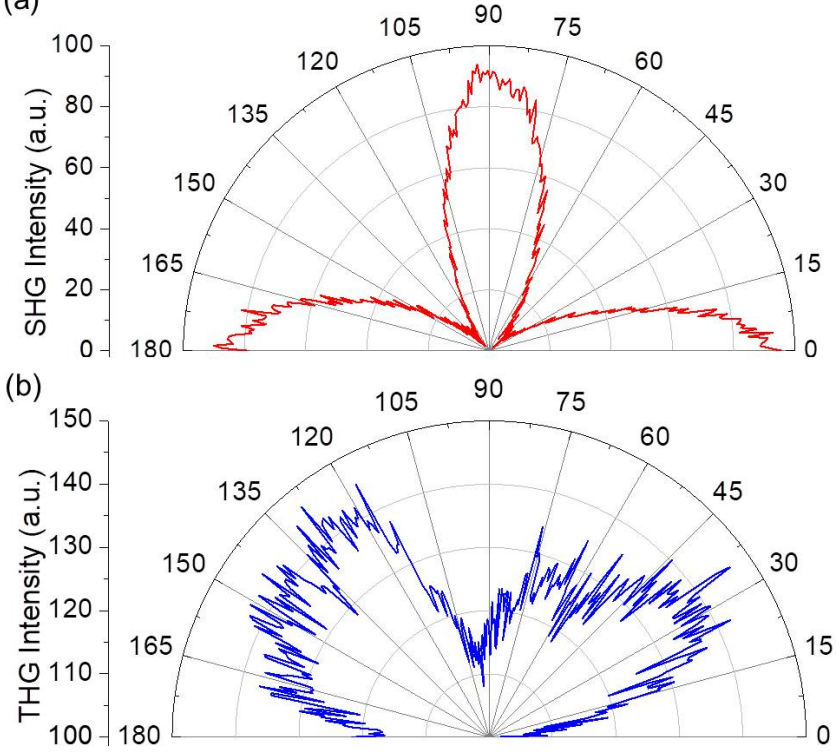

(c)

Angle between incident polarization and HWP optical axis, $\alpha\left({ }^{\circ}\right)$

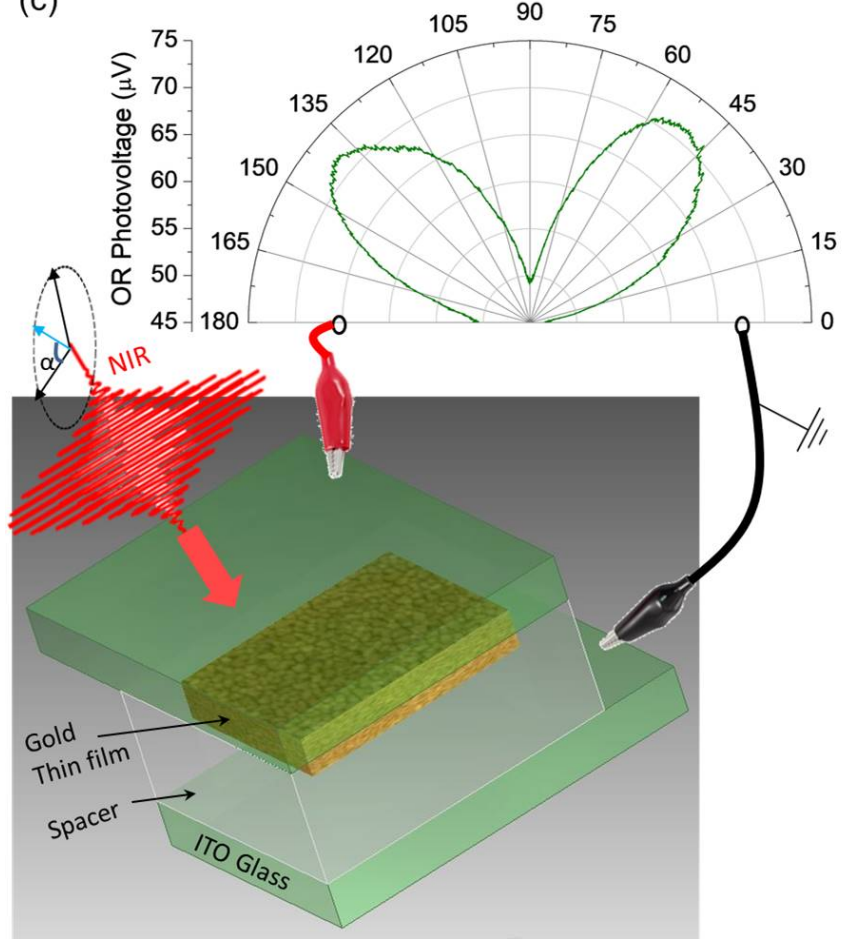

Fig. 6. Polarization dependence of the nonlinear signals generated through a $14 \mathrm{~nm}$ gold nanostructured thin film on top of an ITO substrate. (a) SHG intensity, (b) THG intensity, (c) OR photovoltage and schematic representation of the measurement configuration

The SHG signal is enlarged and diminished while the sample is excited with a wave that is polarized parallel and perpendicular to the incidence plane ( $p$ - and $s$-polarized pump laser), respectively. As is known, in a centrosymmetric material and according to the dipole approximation, the second-order bulk nonlinear susceptibility must vanish [5]. Therefore, the detected SHG signal is due to the broken inversion symmetry at the interface, where the only three nonvanishing independent second-order susceptibility tensor components are: 
$\chi_{z z z}, \chi_{z x x}=\chi_{z y y}$ and $\chi_{x x z}=\chi_{x z x}=\chi_{y y z}=\chi_{y z y}$ [52]. In the illumination configuration shown in Figure 2 and for an s-polarized incident light, the $\chi_{\mathrm{zzz}}$ component, which is the largest surface nonzero second-order susceptibility element [44] doesn't contribute to the SHG emission.

Unlike the SHG signal, which only arises in non-centrosymmetric materials, the THG is allowed in all materials, since the third-order susceptibility $\chi^{(3)}$ is non-vanishing regardless of the symmetry of materials. There are 21 nonzero elements left for the third order nonlinear susceptibility tensor of an isotropic media from which only three are independent. The main relation between these nonzero elements is: $\chi_{y y y y}=\chi_{x x x x}=\chi_{z z z z}=\chi_{z z y y}+\chi_{z y z y}+\chi_{z y y z}[53,54]$. So it is expected to get a larger THG signal when the sample is irradiated by an s-polarized light. Interestingly, owing to the non-phase matched conditions of the process, the largest contributions to THG will come from the interfaces [55].

Surprisingly, the DC photovoltage polarization plotted in Fig. 6(c) shows a similar behavior to THG emission, and not to the SHG one. This indicates the SHG and OR signals have different origins in this sample. The process, which can also explain photovoltage enhancement under $s$-polarized illumination, is explained below.

The polarization giving rise to the $\mathrm{OR}$ signal needs not necessarily be second-order in the pump light's electric field. It has been shown that when suitable combinations of frequencies and static fields are used, higher-order nonlinear polarizations can also lead to rectification [56]. In this situation, the rectification process is via a third-order nonlinear optical process in Eq.1 called electric-field-induced optical rectification (EFIOR).

$$
P_{i}^{(0)}=\chi_{i j k z}^{(3)}(0 ; \omega, \omega, D C) E_{j}^{(\omega)} E_{k}^{(\omega)} E_{z}^{D C}
$$

Where $E_{z}^{D C}$, is the DC electric field normal to the surface, proportional to the static depletion field. It has been shown that at the ITO/gold interface, a local depletion forms due to hot-electron injection from gold into the ITO layer [57], which leads to a local rectifying Schottky barrier. Therefore, the features of the DC photovoltage shown in Fig. 6(c) can be explained using the same tensor components as for THG and both are related to the $\chi_{i j k z}^{(3)}$ elements.

We believe that our proposed method of study could reveal the true origin of the detected photovoltage from the metallic nanostructure under intense illumination to be EFIOR, but not the normally expected second order nonlinear OR process, although some contribution from the latter one cannot be ruled out.

\section{CONCLUSIONS}

Production of electrical DC photovoltage through thin film illumination has several applications in energy conversion and broadband photodetection. While there has been ongoing research on the nonlinear optical rectification (OR) process as a source of DC photovoltage generation, evidence in the literature and in our own results suggest that there are other nonlinear processes that may contribute to DC photovoltage generation during OR detection in a capacitor configuration experiments. Herein, we developed techniques to investigate the true origins of the detected DC photovoltage in an OR detection setup. A clear interpretation of induced photovoltage is an asset to further pursue signal enhancement for potential applications. In summary, the induced photovoltage via multiphoton absorption, work function variations and optical rectification processes were studied in similarly designed experiments. Our results suggest a baseline to study the origin of the detected photovoltages under an intense illumination through some characteristic tests such as (1) study the photovoltage scaling with fundamental power, and (2) study the outcomes of linear and circular polarization variations of the light source on the induced photovoltages.

We finally considered the implications of these tests for a metal nanostructure in a capacitor configuration to address the true origin of the detected OR photovoltage, which proved to be different from the expected one.

Ultimately, we believe that advances in the photovoltage generation via the optical rectification process may give rise to additional unprecedented applications in light detection technologies, rectifying antenna photovoltaics, and sensing applications.

APPENDIX A. Organic Photodetectors [58]: The glass slides, coated with a patterned indium tin oxide (ITO) layer of $120-160 \mathrm{~nm}$ with a sheetresistance of $9-15 \Omega /$ sq, were bought from Lumtec. The ITO slide was cleaned with water, isopropanol, and acetone by sonication in an ultrasonic cleaner for 10 minin each solvent. The slide was dried with a stream of nitrogen. A thin film of zinc oxide ( $\mathrm{ZnO}$ ) was used on top of the ITO electrode as the interface layer to create a more efficient charge transfer path. A solution of $0.75 \mathrm{M}$ zinc acetate dihydrate in $0.75 \mathrm{M}$ monoethanolamine in 2-methoxyethanol was stirred for about 12 hours. The solution was then spin-coated on the patterned ITO on a glass slide at $2000 \mathrm{rpm}$ for $40 \mathrm{~s}$ to get a layer thickness of about $40 \mathrm{~nm}$. The slide was then baked on a hot plate for 5 mins at $275^{\circ} \mathrm{C}$, and then rinsed with deionized water, isopropanol, and acetone. The slide was dried under a jet of nitrogen and the solvent was removed by heating at $200{ }^{\circ} \mathrm{C}$ for 5 mins. This slide was then ready to be spin-coated by the active layer of P3HT: PCBM. A solution of P3HT: PCBM (1:1 wt. ratio) in 1,2-dichlorobenzene was stirred for about 12 hours. The solution was then filtered through 0:2 $\mu \mathrm{m}$ PTFE filter to remove any aggregates and stored in a vile. In fact, it is advised to sonicate the solution briefly just before spin-coating the layer. The layer was spin-coated on top of the $\mathrm{ZnO}$ layer at $600 \mathrm{rpm}$ for $1 \mathrm{~min}$, to geta layer thickness of about $250 \mathrm{~nm}$. Following spin-coating, the slide was annealed at $110{ }^{\circ} \mathrm{C}$ for about 10 mins. A $120 \mathrm{~nm}$ thick silver thin film was deposited by vacuum thermal evaporation at a chamber pressure of $\sim 10^{-6}$ Torr and a deposition rate of $1.5 \AA$ /s as the cathode.

APPENDIX B. ITO-Based Capacitor. The ITO glass was cleaned as outlined above. A gold thin film was deposited by vacuum thermal evaporation at a chamber pressure of about $10^{-6}$ Torr via a deposition rate of $1 \AA /$ s for a smooth thin film and $0.3 \AA$ s for a nanostructured thin film. However, the ITO glass substrate was heated at $180^{\circ} \mathrm{C}$ before and during the deposition process inside the vacuum chamber to achieve the appropriate gold nanostructured film. The bottom ITO glass-Au electrode and the top ITO glass electrode were assembled into a sandwich-type cell and sealed with a hot-melt spacer in between the electrodes. The gap between the electrodes was filled with air.

APPENDIX C. Data Acquisition. A schematic of the custom designed optical scanning apparatus is shown in Figure 2. The photovoltage measurements reported in this work were recorded with a Zurich Instruments UHF lock-in amplifier with an input impedance of $1 \mathrm{M} \Omega$. The FemtoFiber Scientific (Toptica) laser source-generating p-polarized light pulses centered at a wavelength of $1550 \mathrm{~nm}$ with a $100 \mathrm{fs}$-duration and a $90-\mathrm{MHz}$ repetition rate was used as the light source. The transmitted light was also collected and guided to the detection setup to measure simultaneously-generated second and third harmonic signals via the Ocean Optics spectrometer. The linear or elliptical polarization states were varied using a polarized laser light followed by a rotatable half or quarter wave plate (HWP or QWP), respectively. 
Funding. Research reported herein was funded by the Natural Science Research Council of Canada Discovery Grants (RGPIN-2015-05485).

1. M. Bass, P. A. Franken, J. F. Ward, and G. Weinreich, "Optical Rectification", Phys. Rev. Lett. 9, 446 (1962).

2. A. Nahata and T. F. Heinz, "Generation of subpicosecond electrical pulses by optical rectification," Opt. Lett. 23, 11, 867 (1998).

3. J. Zhang, J. D. Bull, and T. E. Darcie, "Microwave photonic signal detection using phase-matched optical rectification in an AlGaAs waveguide," IEEE Photon. Technol. Lett. 19(24), 2012-2014 (2007).

4. Y. Uchiho, M. Shimojo and K. Kajikawa, "Electro-optic effect and optical rectification in gold nanoparticles immobilized above a gold surface," J. Phys. D: Appl. Phys. 43, 495101 (2010).

5. R. W. Boyd, "Nonlinear optics," The Institute of Optics, U. of Rochester, 3rd Edition (2008)

6 B. N. Morozov and Y. M. Aivazyan, "Optical rectification effect and its applications (review)," Sov. J. Quantum Electron. 10, 1-16 (1980).

7. J. Zhang, L. Shi, Y. Wang, E. Cassan, X. Zhang, "On-chip high-speed optical detection based on an optical rectification scheme in silicon plasmonic platform," Opt. Express 22, No. 22, 27504-27514 (2014).

8. Z. Zhu, S. Joshi, and G. Moddel, "High performance room temperature rectenna IR detectors using graphene geometric diodes," IEEE 20, 6 (2014).

9. E. Donchev, J. Pang, P. Gammon, A. Centeno, F. Xie, P. Petrov, J. Breeze, M. Ryan, D. Riley, N. Alford, "The rectenna device: From theory to practice," MRS Energy \& Sustainability 1, 1-34 (2014)

10. D. Y. Goswami, S. Vijayaraghavan, S. Lu, and G. Tamm, “New and emerging developments in solar energy," Solar Energy 76, 33-43 (2004).

11. R. Corkish, M. A. Green, T. Puzzer, "Solar energy collection by antennas," Solar Energy 73,395, (2002).

12 J.M. Nunzi, "Requirements for a rectifying antenna solar cell technology," Proc SPIE 7712, 771204 (2010).

13. R. Uzawa, D. Tanaka, H. Okawa, K. Hashimoto, and K. Kajikawa, "Optical rectification in self-assembled monolayers probed at surface plasmon resonance condition," App. Phys. Lett. 95, 021107 (2009).

14. G. M. Mikheev and R. G. Zonov, A. N. Obraztsov, and Yu. P. Svirko, "Giant optical rectification effect in nanocarbon films," App. Phys. Lett. 84, 24 (2004). 15. G. M. Mikheev, R. G. Zonov, A. N. Obraztsov, A. P. Volkov, and Yu. P. Svirko, "Quick-Response Film Photodetector of High-Power Laser Radiation Based on the Optical Rectification Effect," Tech. Phys. 51, 9, 1190-1196 (2006).

16. T. Baehr-Jones, J. Witzens, and M. Hochberg, "Theoretical Study of Optical Rectification at Radio Frequencies in a Slot Waveguide," IEEE J. of Quantum Elec. 46, 11 (2010).

17. S. M.A. Mirzaee, B.S. Rao, J.M. Nunzi, "Three Photon Absorption Detection Using Polymer Photo- Diodes," Proc. SPIE 8915, 891514 (2013).

18. F. Wang and N. A. Melosh, "Plasmonic Energy Collection through Hot Carrier Extraction," Nano Lett., 11, 5426 (2011).

19. J. C. Riviere, "Contact Potential Difference Measurements by the Kelvin Method," Phys. Soc. B 70, 676-686 (1957)

20. B. Ratier, J.M. Nunzi, M. Aldissi, T.M. Kraft, and E. Buncel, "Organic solar cell materials and active layer designs - improvements with carbon nanotubes: a review," Polym. Int. 61, 342-354 (2012)

21. F. Liu, J.M. Nunzi, "Enhanced organic light emitting diode and solar cell performances using silver nano-clusters," Org. Elec. 13, 1623-1632 (2012).

22. S. Deckers, S. Vandendriessche, D. Cornelis, F. Monnaie, G. Koeckelberghs, I. Asselberghs, T. Verbiest and M. A. van der Veen, "Poly(3-alkylthiophene)s show unexpected second-order nonlinear optical response," Chem. Commun. 50, 2741-2743 (2014)

23. Y. Tong, X. Zhao, M. C. Tan, R. Zhao, "Cost-Effective and Highly Photoresponsive Nanophosphor- P3HT Photoconductive Nanocomposite for Near-Infrared Detection," Sci. Rep. 5, 16761 (2015)

24. W. L. Kalb, S. Haas, C. Krellner, T. Mathis, B. Batlogg, "Trap density of states in small-molecule organic semiconductors: A quantitative comparison of thinfilm transistors with single crystals," Phys. Rev. B 81, 155315 (2010).

\section{REFERENCES}

25 S. V. Novikov, A. R. Tameev, A. V. Vannikov, J.M. Nunzi, "Estimation of the concentration of deep traps in organic photoconductors using two-photon absorption," Proc. SPIE 7993, 799321 (2011).

26. R. A. Street, A. Krakaris, and S.R. Cowan, "Recombination Through Different Types of Localized States in Organic Solar Cells," Adv. Funct. Mater. 22,4608 (2012)

27. C. Sentein, C. Fiorini, A. Lorin, L. Sicot, J.M. Nunzi, "Study of orientation induced molecular rectification in polymer films", Opt. Mat. 9, 316-322 (1998). 28. M. Akbi, A. Lefort," Work function measurements of contact materials for industrial use," J. Phys. D: Appl. Phys. 31, 1301-1308 (1998).

29. W. M. H. Sachtler, G. J. H. Dorgelo and A. A. Holscher, "The Work Function of Gold," Surf. Sci. 5, 221-229 (1966).

30. D. Burgess Jr., P. C. Stair, and E. Weitz "Calculations of the surface temperature rise and desorption temperature in laser-induced thermal desorption," J. Vac. Sci. Technol. A 4, 3 (1986).

31. J. R. Trantum, M. L. Baglia, Z. E. Eagleton, R. L. Mernaugh, and F. R. Haselton, "Biosensor design based on Marangoni flow in an evaporating drop, Lab Chip," 14, 315 (2014).

32. J.P. Bourgoin, G.G. Allogho, and A. Haché, "Thermal conduction in thin films measured by optical surface thermal lensing," J. of App. Phys. 108, 073520 (2010).

33. T. Kemp, T. A. S. Srinivas, R. Fettig, and W. Ruppel, "Measurement of thermal diffusivity of thin films and foils using a laser scanning microscope," Rev. of Sci. Instruments 66, 176 (1995).

34. G. Chen, P. Hui, "Thermal conductivities of evaporated gold films on silicon and glass," Appl. Phys. Lett. 74, 2942 (1999).

35. P. B. Johnson and R. W. Christy, "Optical constants of the noble metals," Phys. Rev. B 6, 4370-4379 (1972).

36. M. Akbi, "A Method for Measuring the Photoelectric Work Function of Contact Materials Versus Temperature," IEEE Trans. Compon. Packag. Manuf. Technol. 4, 8, 1293-1302 (2014)

37. A. N. Obraztsov, et al. "Photon drag effect in carbon nanotube yarns," App. Phys. Lett. 94, 231112 (2009).

38. A. S. Vengurlekar, and T. Ishihara, "Surface plasmon enhanced photon drag in metal films," App. Phys. Lett. 87, 091118 (2005).

39. F. Nastos, J. E. Sipe, "Optical rectification and current injection in unbiased semiconductors," Phys. Rev. B 82, 235204 (2010).

40. V. L. Malevich, R. Adomavicius, and Krotkus, A., "THZ emission from semiconductor surfaces," C. R. Phys. 9, 130, 141 (2008).

41 G. Klatt1, F. Hilser, W. Qiao, M. Beck, R. Gebs, A. Bartels, K. Huska, U. Lemmer, G. Bastian, M.B. Johnston, M. Fischer, J. Faist, T. Dekorsy, "Terahertz emission from lateral photo-Dember currents," Opt. Exp. 4934, 18, 5 (2010).

42. M. Chen, J. Gu, C. Sun, Y. Zhao, R. Zhang, X. You, Q. Liu, W. Zhang, Y. Su, H. $\mathrm{Su}$, and D. Zhang "Light-Driven Overall Water Splitting Enabled by a PhotoDember Effect Realized on 3D Plasmonic Structures," ACS Nano 10, 7, 6693 6701 (2016)

43. J. E. Sipe, V. C. Y. So, M. Fukui, and G. I. Stegeman, "Analysis of second harmonic generation at metal surfaces," Phys. Rev. B Vol. 21, 10 (1980).

44. F. X. Wang, F. J. Rodriguez, W. M. Albers, R. Ahorinta, J. E. Sipe, M. Kauranen, "Surface and bulk contributions to the second-order nonlinear optical response of a gold film," Phys. Rev. B80, 233402 (2009).

45. G. Ramakrishnan, and P. C. M. Planken, "Percolation-enhanced generation of terahertz pulses by optical rectification on ultrathin gold films," Opt. Lett. 36, 13 (2011)

46. T. Vicsek, Fractal Growth Phenomena, (World Sc.: Singapore 1992).

47. R. F. Voss, R. B. Laibowitz, and E. I. Allessandrini, "Fractal (Scaling) Clusters in Thin Gold Films near the Percolation Threshold," Phys. Rev. Lett. 49, 1441-1444 (1982) 
48. H. Wei, and H. Eilers, "From silver nanoparticles to thin films: Evolution of microstructure and electrical conduction on glass substrates," J. Phys. Chem. Solids 70, 459-465 (2009).

49. X. Liu, D. Li, X. Sun, Z. Li, H. Song, H. Jiang, and Y. Chen, "Tunable Dipole Surface Plasmon Resonances of Silver Nanoparticles by Cladding Dielectric Layers," Sci. Rep. 5, 12555 (2015).

50. S. M.A. Mirzaee, O. Lebel, J.M. Nunzi, "Simple Unbiased Hot-Electron Polarization-Sensitive Near-Infrared Photodetector," ACS Appl. Mater. Interfaces 10, 14, 11862-11871 (2018).

51. V. M. Shalaev, Nonlinear Optics of Random Media: Fractal Composites and Metal-Dielectric Films, (Springer Science \& Business Media: Las Cruces, NM, 1999).

52. D. Krause, C.W. Teplin, and C.T. Rogers, "Optical surface second harmonic measurements of isotropic thin-film metals: Gold, silver, copper, aluminum, and tantalum," J. Appl. Phys. 96, 7 (2004).
53 N. Olivier, F. Aptel, K. Plamann, M. Schanne-Klein, and E. Beaurepaire, "Harmonic microscopy of isotropic and anisotropic microstructure of the human cornea," Opt. Exp. 18, 5, 5028 (2010).

54 P.-F. Brevet, Surface Second Harmonic Generation, (PPUR presses polytechniques, p.47. 1997).

55 R. Barille, L. Canioni, L. Sarger, and G. Rivoire, "Nonlinearity measurements of thin films by third-harmonic-generation microscopy," Phys. Rev. E 66, 067602 (2002).

56. E. Yablonovitch, J. P. Heritage, D. E. Aspnes, and Y. Yafet, "Virtual photoconductivity," Phys. Rev. Lett. 63, 976-979 (1989).

57. M. Abb, P. Albella, J. Aizpurua, and O. L. Muskens, "All-Optical Control of a Single Plasmonic Nanoantenna-ITO Hybrid” Nano Lett. 11, 2457-2463 (2011). 58 T. Adhikari, Z. G. Rahami, J. M. Nunzi, and O. Lebel, "Synthesis, characterization and photovoltaic performance of novel glass-forming perylenediimide derivatives," Org. Elec. 34, 146-156 (2016). 